\title{
Incremental Synthesis of Fault-Tolerant Real-Time Programs $^{1}$
}

\author{
Borzoo Bonakdarpour and Sandeep S. Kulkarni \\ Department of Computer Science and Engineering \\ Michigan State University \\ East Lansing, MI 48824, USA \\ Email: $\{$ borzoo, sandeep $\}$ @ cse.msu.edu \\ http://www.cse.msu.edu/ $\{$ borzoo, sandeep $\}$
}

\begin{abstract}
In this paper, we focus on the problem of automated addition of faulttolerance to an existing fault-intolerant real-time program. We consider three levels of fault-tolerance, namely nonmasking, failsafe, and masking, based on safety and liveness properties satisfied in the presence of faults. More specifically, a nonmasking (respectively, failsafe, masking) program satisfies liveness (respectively, safety, both safety and liveness) in the presence of faults. For failsafe and masking fault-tolerance, we consider two additional levels, soft and hard, based on satisfaction of timing constraints in the presence of faults. We present a polynomial time algorithm (in the size of the input program's region graph) that adds bounded-time recovery from an arbitrary given set of states to another arbitrary set of states. Using this algorithm, we propose a sound and complete synthesis algorithm that transforms a fault-intolerant real-time program into a nonmasking fault-tolerant program. Furthermore, we introduce sound and complete algorithms for adding soft/hard-failsafe fault-tolerance. For reasons of space, our results on addition of soft/hard-masking fault-tolerance are presented in a technical report.
\end{abstract}

Keywords: Fault-tolerance, Real-time, Bounded-time recovery, Program synthesis, Program transformation, Formal methods.

\section{Introduction}

Automated program synthesis is the problem of designing an algorithmic method to find a program that satisfies a required set of properties. Such automated synthesis is desirable, as it ensures that the synthesized program is correct-by-construction. In existing specification-based synthesis methods, a change in the specification requires us to redo synthesis from scratch. Thus, it would be advantageous, if we could reuse the previous

\footnotetext{
${ }^{1}$ This work was partially sponsored by NSF CAREER CCR-0092724, DARPA Grant OSURS01-C-1901, ONR Grant N00014-01-1-0744, NSF grant EIA-0130724, and a grant from Michigan State University.
} 
efforts made to synthesize real-time programs and somehow incrementally add properties (e.g., fault-tolerance) to them. Moreover, such incremental synthesis is especially useful if the given real-time program is designed manually, e.g., for ensuring that the original program is efficient. More importantly, incremental synthesis is crucial when the existing real-time program satisfies properties whose automated synthesis is undecidable (e.g., precise eventuality $\nabla_{=\delta} q$ ) or lies in highly complex classes of complexity.

In this paper, we focus on designing incremental synthesis algorithms that solely add fault-tolerance to existing fault-intolerant real-time programs, where processes can read and write all program variables in one atomic step. In particular, we concentrate on algorithms with manageable time and space complexity such that they can be used in tools for synthesizing fault-tolerant real-time programs. To characterize such manageable complexity, we require that the complexity of our algorithms are comparable to that of existing model checking techniques in the dense real-time model.

In order to characterize fault-tolerance requirements of programs, in our work, we consider three levels of fault-tolerance, namely nonmasking (respectively, stabilizing), failsafe, and masking, based on safety and liveness properties satisfied in the presence of faults. Furthermore, we propose two additional levels, namely soft and hard faulttolerance, based on satisfaction of timing constraints in the presence of faults. Precisely, in the absence of faults, both soft and hard fault-tolerant programs are required to satisfy their timing constraints. However, in the presence faults, a soft fault-tolerant program is not required to satisfy its timing constraints while a hard fault-tolerant program is required to do so. In this sense, for instance, a hard-failsafe program satisfies its safety specification as well as its timing constraints in the presence of faults.

\subsection{Related Work}

In the literature of real-time computing, fault-tolerance has mostly been addressed in the context of scheduling theory (e.g., $[1,2])$. In fault-tolerant real-time scheduling, the objective is to find the optimal schedule of a set of tasks on a set of processors dynamically, such that the largest possible number of tasks meet their deadlines. Since time complexity is a critical issue in dynamic scheduling, most of the proposed algorithms are in the form of heuristics designed for specific platforms and special types of faults (e.g., transient, fail-stop, Byzantine, etc.).

Recently, we studied the problem of incremental synthesis of timed automata in the absence of faults in [3]. More specifically, we developed synthesis algorithms and hardness results for adding different types of bounded response properties to a given timed automaton. We also studied the problem of incremental addition of UNITY [4] properties to untimed programs in [5].

The problem of synthesizing untimed fault-tolerant programs has been studied in the literature from different perspectives. In [6-8], the authors propose synthesis methods for adding fault-tolerance and multitolerance to existing untimed programs. In [9], Attie, Arora, and Emerson study the problem of synthesizing fault-tolerant concurrent untimed programs from temporal logic specifications expressed in CTL formulas.

Synthesis of real-time systems has mostly been studied in the context of controller synthesis and game theory [10-15]. In these papers, the common assumption is that the existing program (called a plant) and/or the given specification are deterministic. 
Moreover, since the authors consider highly expressive specifications, the complexity of proposed methods is very high. For example, synthesis problems presented in $[10,11$, $14,15]$ are EXPTIME-complete. Moreover, deciding the existence of a solution (called a controller) in $[12,13]$ is 2EXPTIME-complete.

\subsection{Contributions}

In this paper we (i) introduce a generic fault-tolerance framework for real-time programs independent of platform, architecture, and type of faults; (ii) extend the previous work by Kulkarni and Arora [6] to the context of real-time programs; (iii) consider a general notion of real-time programs that covers both deterministic and nondeterministic programs in both synchronous and asynchronous models; and (iv) introduce various levels of fault-tolerance for real-time systems based on satisfaction of properties and timing constraints in the presence of faults. Furthermore, we present a class of specifications where we can express typical requirements for specifying real-time and fault-tolerant systems and we show that the complexity of synthesis algorithms for this class of specifications is comparable to existing model checking techniques for real-time programs [16]. Moreover, since we follow the standard model of timed automata [17], many of the problems in fault-tolerant scheduling theory can be modeled in our framework [18].

The main results in this paper are as follows. First, we present a polynomial time algorithm (in the size of the input program's region graph) that adds bounded-time recovery from an arbitrary given set of states to another arbitrary set of states. Then, using this algorithm, we propose sound and complete synthesis algorithms that transform a faultintolerant real-time program into a (1) nonmasking or soft-failsafe fault-tolerant programs, or (2) hard-failsafe fault-tolerant program where the synthesized fault-tolerant program is required to satisfy at most one bounded response property in the presence of faults. For reasons of space, in a technical report [19], we also present a synthesis algorithm for adding soft-masking fault-tolerance. Moreover, we show that the problem of adding hard-masking fault-tolerance where the synthesized program is required to satisfy at least two bounded response properties in the presence of faults is NP-hard.

Organization of the paper. In Section 2, we present formal definitions of real-time programs, specifications, and regions graphs. We introduce the notions of faults and fault-tolerance in the context of real-time programs in Section 3. In Section 4, we formally state the problem of adding fault-tolerance to real-time programs. We present our synthesis algorithms for adding nonmasking, soft-failsafe, and hard-failsafe faulttolerance in sections 5, 6, and 7, respectively. Finally, in Section 8, we make the concluding remarks.

\section{Real-Time Programs, Specifications, and Region Graphs}

In our framework, programs are specified in terms of their state space and their transitions [20]. The definition of specifications is adapted from Henzinger [21]. Finally, the notion of region graph is due to Alur and Dill [17]. 


\subsection{Real-Time Program}

Let $V$ be a finite set of discrete variables and $X$ be a finite set of clock variables. Each discrete variable is associated with a finite domain $D$ of values. A location is a function that maps each discrete variable to a value from its respective domain. A clock constraint over the set $X$ of clock variables is a Boolean combination of formulas of the form $x \preceq c$ or $x-y \preceq c$, where $x, y \in X, c \in \mathbb{Z}_{\geq 0}$, and $\preceq$ is either $<$ or $\leq$. We denote the set of all clock constraints over $X$ by $\Phi(X)$. A clock valuation is a function $\nu: X \rightarrow \mathbb{R}_{\geq 0}$ that assigns a real value to each clock variable. Furthermore, for $\tau \in \mathbb{R}_{\geq 0}, \nu+\tau=\nu(x)+\tau$ for every clock $x$. Also, for $\lambda \subseteq X, \nu[\lambda:=0]$ denotes the clock valuation for $\mathrm{X}$ which assigns 0 to each $x \in \lambda$ and agrees with $\nu$ over the rest of the clock variables in $X$.

A state (denoted $\sigma$ ) is a pair $(s, \nu)$, such that $s$ is a location and $\nu$ is a clock valuation for $X$ at location $s$. A transition (denoted $\left.\left(\sigma_{0}, \sigma_{1}\right)\right)$ is of the form $\left(s_{0}, \nu_{0}\right) \rightarrow\left(s_{1}, \nu_{1}\right)$. Transitions are classified into two types:

- Delay: for a state $\sigma=(s, \nu)$ and a time duration $\delta \in \mathbb{R}_{\geq 0}$ (denoted $(\sigma, \delta)$ ), $(s, \nu) \rightarrow(s, \nu+\delta)$.

- Jump: for a state $\left(s_{0}, \nu\right)$, a location $s_{1}$, and a set $\lambda$ of clock variables, $\left(s_{0}, \nu\right) \rightarrow$ $\left(s_{1}, \nu[\lambda:=0]\right)$.

A program $\mathcal{P}$ is a tuple $\left\langle S_{p}, \psi_{p}\right\rangle$, where $S_{p}$ is the state space, and $\psi_{p}$ is a set of transitions. Let $\psi_{p}^{s}$ and $\psi_{p}^{d}$ denote the set of jump and delay transitions in $\psi_{p}$, respectively. A state predicate is a subset of $S_{p}$ such that it is definable by the above syntax of clock constraints, i.e., in the corresponding Boolean expression clock variables are only compared to nonnegative integers. A state predicate $S$ is closed in program $\mathcal{P}$ if $\left(\left(\forall\left(\sigma_{0}, \sigma_{1}\right) \in \psi_{p}^{s}:\left(\sigma_{0} \in S \Rightarrow \sigma_{1} \in S\right)\right) \wedge\left(\forall(\sigma, \delta) \in \psi_{p}^{d}:(\sigma \in S \Rightarrow \forall \epsilon \leq\right.\right.$ $\delta: \sigma+\epsilon \in S)))$. A timed state sequence $\left\langle\left(\sigma_{0}, \tau_{0}\right),\left(\sigma_{1}, \tau_{1}\right), \cdots\right\rangle$, where $\tau_{i} \in \mathbb{R}_{\geq 0}$, is a computation of $\mathcal{P}$ if the following conditions are satisfied: (1) $\forall j>0:\left(\sigma_{j-1}, \sigma_{j}\right) \in \psi_{p}$, (2) if it is finite and terminates in $\left(\sigma_{l}, \tau_{l}\right)$ then there does not exist state $\sigma$ such that $\left(\sigma_{l}, \sigma\right) \in \psi_{p}$, and (3) the sequence $\left\langle\tau_{0}, \tau_{1}, \cdots\right\rangle$ satisfies the following constraints:

Monotonicity: $\tau_{i} \leq \tau_{i+1}$ for all $i \in \mathbb{N}$.

Divergence: For all $t \in \mathbb{R}_{\geq 0}$, there exists $j$ such that $\tau_{j} \geq t$.

The projection of a set of program transitions $\psi_{p}$ on state predicate $S$ (denoted $\psi_{p} \mid S$ ) is the set of transitions $\left\{\left(\sigma_{0}, \sigma_{1}\right) \in \psi_{p}^{s} \mid \sigma_{0}, \sigma_{1} \in S\right\} \cup\left\{(\sigma, \delta) \in \psi_{p}^{d} \mid \sigma \in S \wedge(\forall \epsilon \leq\right.$ $\delta: \sigma+\epsilon \in S)\}$.

\subsection{Specification}

A specification (or property), denoted $\Sigma$, is a set of timed state sequences of the form $\left\langle\left(\sigma_{0}, \tau_{0}\right),\left(\sigma_{1}, \tau_{1}\right), \cdots\right\rangle$. Following Henzinger [21], we require that the sequence $\left\langle\tau_{0}, \tau_{1}, \cdots\right\rangle$ satisfies monotonicity and divergence. We now define what it means for a program $\mathcal{P}$ to satisfy a specification $\Sigma$. Given a program $\mathcal{P}$, a state predicate $S$, and a specification $\Sigma$, we write $\mathcal{P} \models_{S} \Sigma$ and say that program $\mathcal{P}$ satisfies $\Sigma$ from $S$ iff (1) 
$S$ is closed in $\mathcal{P}$, and (2) every computation of $\mathcal{P}$ that starts where $S$ is true is in $\Sigma$. If $\mathcal{P} \models_{S} \Sigma$ and $S \neq\{\}$, we say that $S$ is an invariant of $\mathcal{P}$ for $\Sigma$.

Notation. Whenever the specification is clear from the context, we will omit it; thus, " $S$ is an invariant of $\mathcal{P}$ " abbreviates " $S$ is an invariant of $\mathcal{P}$ for $\Sigma$ ".

We say that program $\mathcal{P}$ maintains $\Sigma$ iff for all finite timed state sequences $\alpha$ of $\mathcal{P}$, there exists a timed state sequence $\beta$ such that $\alpha \beta \in \Sigma$. We say that $\mathcal{P}$ violates $\Sigma$ iff it is not the case that $\mathcal{P}$ maintains $\Sigma$. Note that, the definition of maintains identifies the property of finite timed state sequences, whereas the definition of satisfies expresses the property of infinite timed state sequences.

Following Alpern and Schneider [22] and Henzinger [21], we let the specification consist of a liveness specification and a safety specification. The liveness specification is represented by a set of infinite computations. A program satisfies the liveness specification, if every computation prefix of the program has a suffix that is in the liveness specification.

Remark 2.1: In the synthesis problem, we begin with an initial fault-intolerant program that satisfies its specification (including the liveness specification) in the absence of faults. We will show that our synthesis algorithms preserve liveness specification. Hence, the liveness specification need not be specified explicitly.

In this paper, with abuse of notation, we let the safety specification consist of (1) a set $\Sigma_{b t}$ of bad transitions that should not occur in the program computation, i.e., a subset of $\left\{\left(\sigma_{0}, \sigma_{1}\right) \mid \sigma_{0}, \sigma_{1} \in S_{p}\right\}$, and (2) a conjunction of zero or more bounded response properties of the form $\Sigma_{b r} \equiv\left(\left(P_{1} \mapsto \leq \delta_{1} Q_{1}\right) \wedge\left(P_{2} \mapsto \leq \delta_{2} Q_{2}\right) \wedge \ldots \wedge\left(P_{m} \mapsto \leq \delta_{m}\right.\right.$ $\left.Q_{m}\right)$ ), i.e., it is always the case that a state in $P_{i}$ is followed by a state in $Q_{i}$ within $\delta_{i}$ time units, where $P_{i}$ and $Q_{i}$ are state predicates and $\delta_{i} \in \mathbb{Z}_{\geq 0}$, for all $i$ such that $1 \leq i \leq m$. Observe that we abuse the $\models$ notation for the set $\Sigma_{b t}$ of bad transitions. This is because it is possible to trivially translate this concise representation of safety into the corresponding set of infinite computations. The same concept applies to definitions of maintains and violates.

\subsection{Region Graph}

Real-time programs can be analyzed with the help of an equivalence relation of finite index on the set of states [17]. Given a real-time program $\mathcal{P}$, for each clock $x \in X$, let $c_{x}$ be the largest constant in the guards of transitions and invariant of $\mathcal{P}$ that involve $x$, where $c_{x}=0$ if $x$ does not occur in any guard or invariant of $\mathcal{P}$. Two clock valuations $\nu, \mu$ are clock equivalent if (1) for all $x \in X$, either $\lfloor\nu(x)\rfloor=\lfloor\mu(x)\rfloor$ or both $\nu(x), \mu(x)>c_{x}$, (2) the ordering of the fractional parts of the clock variables in the set $\left\{x \in X \mid \nu(x)<c_{x}\right\}$ is the same in $\mu$, and (3) for all $x \in\left\{y \in X \mid \nu(y)<c_{y}\right\}$, the clock value $\nu(x)$ is an integer if and only if $\mu(x)$ is an integer. A clock region $\rho$ is a clock equivalence class. Two states $\left(s_{0}, \nu_{0}\right)$ and $\left(s_{1}, \nu_{1}\right)$ are region equivalent, written $\left(s_{0}, \nu_{0}\right) \equiv\left(s_{1}, \nu_{1}\right)$, if (1) $s_{0}=s_{1}$ and (2) $\nu_{0}$ and $\nu_{1}$ are clock equivalent. A region is an equivalence class with respect to $\equiv$. Using the region equivalence relation, we construct the region graph of $\mathcal{P}\left\langle S_{p}, \psi_{p}\right\rangle$ (denoted $R(\mathcal{P})\left\langle S_{p}^{r}, \psi_{p}^{r}\right\rangle$ ) as follows. Vertices of $R(\mathcal{P})$ (denoted $S_{p}^{r}$ ) are regions. Edges of $R(\mathcal{P})$ (denoted $\psi_{p}^{r}$ ) are of the form $\left(s_{0}, \rho_{0}\right) \rightarrow\left(s_{1}, \rho_{1}\right)$ iff for some clock valuations $\nu_{0} \in \rho_{0}$ and $\nu_{1} \in \rho_{1}$, $\left(s_{0}, \nu_{0}\right) \rightarrow\left(s_{1}, \nu_{1}\right)$ is a transitions in $\psi_{p}$. We say that a region $\left(s_{0}, \rho_{0}\right)$ of region graph 
$R(\mathcal{P})$ is a deadlock region iff for all regions $\left(s_{1}, \rho_{1}\right)$, there does not exist an edge of the form $\left(s_{0}, \rho_{0}\right) \rightarrow\left(s_{1}, \rho_{1}\right)$. A region predicate $S^{r}$ with respect to a state predicate $S$ is defined by $S^{r}=\{(s, \rho) \mid \exists(s, \nu):((s, \nu) \in S \wedge \nu \in \rho)\}$. Likewise, the region predicate with respect to invariant $S$ of a program $\mathcal{P}$ is called region invariant $S^{r}$. The projection of a set of edges $\psi_{p}^{r}$ on region predicate $S^{r}$ (denoted $\psi_{p}^{r} \mid S^{r}$ ) is the set of edges $\left\{\left(r_{0}, r_{1}\right) \in \psi_{p}^{r} \mid r_{0}, r_{1} \in S^{r}\right\}$.

Region graphs are time-abstract bisimulation of real-time programs [17]. In our synthesis algorithms in section 5, 6, and 7, we transform a real-time program $\mathcal{P}\left\langle S_{p}, \psi_{p}\right\rangle$ into its corresponding region graph $R(\mathcal{P})\left\langle S_{p}^{r}, \psi_{p}^{r}\right\rangle$ by invoking the procedure ConstructRegionGraph. We also let this procedure take state predicates and sets of transitions in $\mathcal{P}$ (e.g., $S$ and $\Sigma_{b t}$ ) and return the corresponding region predicates and sets of edges in $R(\mathcal{P})$ (e.g., $S^{r}$ and $\Sigma_{b t}^{r}$ ). Likewise, we transform a region graph $R(\mathcal{P})$ back to a real-time program by invoking the procedure ConstructRealTimeProgram.

A clock region $\beta$ is a time-successor of a clock region $\alpha$ iff for each $\nu \in \alpha$, there exists $\tau \in \mathbb{R}_{\geq 0}$, such that $\nu+\tau \in \beta$, and $\nu+\tau^{\prime} \in \alpha \cup \beta$ for all $\tau^{\prime}<\tau$. We call a region $(s, \rho)$ a boundary region, if for each $\nu \in \rho$ and for any $\tau \in \mathbb{R}_{\geq 0}, \nu$ and $\nu+\tau$ are not equivalent. A region is open, if it is not a boundary region. A region $(s, \rho)$ is called an end region, if $\nu(x)>c_{x}$ for all $\nu \in \rho$ and for all clocks $x \in X$.

\section{Faults and Fault-Tolerance in Real-Time Programs}

In this section, we extend formal definitions of faults and fault-tolerance due to Arora and Gouda [23] to the context of real-time programs. The faults that a program is subject to are systematically represented by transitions. A class of faults $f$ for program $\mathcal{P}\left\langle S_{p}, \psi_{p}\right\rangle$ is a subset of the set $S_{p} \times S_{p}$. Faults are also categorized into delay faults and jump faults. We use $\psi_{p}[] f$ to denote the transitions obtained by taking the union of the transitions in $\psi_{p}$ and the transitions in $f$.

We say that a state predicate $T$ is an $f$-span (read as fault-span) of $\mathcal{P}$ from $S$ iff the following conditions are satisfied: (1) $S \subseteq T$, and (2) $T$ is closed in $\psi_{p}[] f$. Observe that for all computations of $\mathcal{P}$ that start from states where $S$ is true, $T$ is a boundary in the state space of $\mathcal{P}$ up to which (but not beyond which) the state of $\mathcal{P}$ may be perturbed by the occurrence of the transitions in $f$. As we defined the computations of $\mathcal{P}$, we say that a timed state sequence, $\left\langle\left(\sigma_{0}, \tau_{0}\right),\left(\sigma_{1}, \tau_{1}\right), \cdots\right\rangle$, is a computation of $\mathcal{P}$ in the presence of $f$ iff the following four conditions are satisfied: (1) $\forall j>0:\left(\sigma_{j-1}, \sigma_{j}\right) \in\left(\psi_{p} \cup f\right)$, (2) if it is finite and terminates in state $\left(\sigma_{l}, \tau_{l}\right)$ then there does not exist state $\sigma$ such that $\left(\sigma_{l}, \sigma\right) \in \psi_{p}$, (3) $\left\langle\tau_{0}, \tau_{1}, \cdots\right\rangle$ satisfies monotonicity and divergence, and (4) $\exists n \geq 0$ : $\left(\forall j>n:\left(\sigma_{j-1}, \sigma_{j}\right) \in \psi_{p}\right)$.

We consider three levels of fault-tolerance, namely nonmasking, failsafe, and masking based on satisfaction of safety and liveness properties in the presence of faults. For failsafe and masking fault-tolerance, we propose two additional levels, namely soft and hard, based on satisfaction of timing constraints in the presence of faults. Intuitively, a soft fault-tolerant real-time program is not required to satisfy its timing constraints in the presence of faults. A hard fault-tolerant real-time program must satisfy its timing constraints even in the presence of faults. 
Let specification $\Sigma$ consist of $\Sigma_{b t}$ and $\Sigma_{b r}$. Since a nonmasking fault-tolerant program need not satisfy safety in the presence of faults, $\mathcal{P}$ is nonmasking $f$-tolerant from $S$ for $\Sigma$ with recovery time $\delta$, where $\delta \in \mathbb{Z}_{\geq 0}$, iff (1) $\mathcal{P} \models_{S} \Sigma_{b t}$, (2) $\mathcal{P} \models_{S} \Sigma_{b r}$, and (3) there exists $T$ such that $T$ is an $f$-span of $\mathcal{P}$ from $S$, and every computation of $\mathcal{P}\left\langle S_{p}, \psi_{p}[] f\right\rangle$ that starts from a state in $T$, reaches a state in $S$ within $\delta$ time units. We say that $\mathcal{P}$ is soft-failsafe $f$-tolerant from $S$ for $\Sigma$ iff (1) $\mathcal{P} \models_{S} \Sigma_{b t}$, (2) $\mathcal{P} \models_{S} \Sigma_{b r}$, and (3) there exists $T$ such that $T$ is an $f$-span of $\mathcal{P}$ from $S$, and $\mathcal{P}\left\langle S_{p}, \psi_{p}[] f\right\rangle$ maintains $\Sigma_{b t}$ from $T$. A program $\mathcal{P}$ is hard-failsafe f-tolerant from $S$ for $\Sigma$ iff $\mathcal{P}$ is soft-failsafe $f$-tolerant from $S$ for $\Sigma$ and $\mathcal{P}\left\langle S_{p}, \psi_{p}[] f\right\rangle$ maintains $\Sigma_{b r}$ from $T$. A program $\mathcal{P}\left\langle S_{p}, \psi_{p}\right\rangle$ is soft-masking $f$-tolerant from $S$ for $\Sigma$ with recovery time $\delta$, where $\delta \in \mathbb{Z}_{\geq 0}$, iff (1) $\mathcal{P} \models_{S} \Sigma_{b t},(2) \mathcal{P} \models_{S} \Sigma_{b r},(3)$ there exists $T$ such that $T$ is an $f$-span of $\mathcal{P}$ from $S$ and $\mathcal{P}\left\langle S_{p}, \psi_{p}[] f\right\rangle$ maintains $\Sigma_{b t}$ from $T$, and (4) every computation of $\mathcal{P}\left\langle S_{p}, \psi_{p}[] f\right\rangle$ that starts from a state in $T$, reaches a state in $S$ within $\delta$ time units. A program $\mathcal{P}\left\langle S_{p}, \psi_{p}\right\rangle$ is hard-masking $f$-tolerant from $S$ for $\Sigma$ with recovery time $\delta$, where $\delta \in \mathbb{Z}_{\geq 0}$, iff $\mathcal{P}$ is soft-masking $f$-tolerant from $S$ for $\Sigma$ with recovery time $\delta$, and $\mathcal{P}\left\langle S_{p}, \psi_{p}[] f\right\rangle$ maintains $\Sigma_{b r}$ from $T$.

Notation. Whenever the specification $\Sigma$ and the invariant $S$ are clear from the context, we omit them; thus, " $f$-tolerant" abbreviates " $f$-tolerant from $S$ for $\Sigma$ ".

Assumption 3.1: Since $\mathcal{P}$ satisfies $\Sigma_{b r} \equiv\left(\left(P_{1} \mapsto \leq \delta_{1} Q_{1}\right) \wedge \ldots \wedge\left(P_{m} \mapsto \leq \delta_{m} Q_{m}\right)\right)$ in the absence of faults (cf. Remark 2.1), without loss of generality, we assume that for each bounded response property $\left(P_{i} \mapsto \leq \delta_{i} Q_{i}\right)$, where $1 \leq i \leq m$, the intolerant program already has a clock variable that is reset on transitions that go from a state in $\neg P_{i}$ to a state in $P_{i}$ to keep track of time as soon as $P_{i}$ becomes true.

Assumption 3.2: We assume that faults are immediately detectable and that given a state of the program, we can determine the number of faults that have occurred in reaching that state. This assumption is needed only for addition of hard fault-tolerance and is realistic in many commonly considered systems. For instance, in multiprocessor scheduling theory, a processor-crash is immediately detectable and its number of occurrences is easily traceable.

Assumption 3.3: We assume that the number of occurrence of faults in a program computation is bounded by a pre-specified value $n$. This assumption is required since for commonly considered faults, it can be shown that bounded-time recovery in the presence of unbounded occurrence of faults is impossible.

\section{Problem Statement}

Given are a fault-intolerant real-time program $\mathcal{P}\left\langle S_{p}, \psi_{p}\right\rangle$, its invariant $S$, a set of faults $f$, and a safety specification $\Sigma$ such that $\mathcal{P} \models_{S} \Sigma$. Our goal is to synthesize a realtime program $\mathcal{P}^{\prime}\left\langle S_{p}, \psi_{p}^{\prime}\right\rangle$ with invariant $S^{\prime}$ such that $\mathcal{P}^{\prime}$ is $f$-tolerant from $S^{\prime}$ for $\Sigma$. As mentioned in the introduction, our synthesis methods obtain $\mathcal{P}^{\prime}$ from $\mathcal{P}$ by adding fault-tolerance alone to $\mathcal{P}$, i.e., $\mathcal{P}^{\prime}$ does not introduce new behaviors to $\mathcal{P}$ when no faults have occurred. Observe that:

1. If $S^{\prime}$ contains states that are not in $S$ then, in the absence of faults, $\mathcal{P}^{\prime}$ may include computations that start outside $S$. Since we require that $\mathcal{P}^{\prime} \models_{S^{\prime}} \Sigma$, it would imply that $\mathcal{P}^{\prime}$ is using a new way to satisfy $\Sigma$ in the absence of faults. 
2. If $\psi_{p}^{\prime} \mid S^{\prime}$ contains a transition that is not in $\psi_{p} \mid S^{\prime}$ then $\mathcal{P}^{\prime}$ can use this transition in order to satisfy $\Sigma$ in the absence of faults.

Thus, the synthesis problem is as follows (we instantiate this problem for soft/hardfailsafe, nonmasking, and soft/hard-masking $f$-tolerance in the obvious way):

Problem Statement 4.1. Given $\mathcal{P}\left\langle S_{p}, \psi_{p}\right\rangle, S, \Sigma$, and $f$ such that $\mathcal{P} \models_{S} \Sigma$. Identify $\mathcal{P}^{\prime}\left\langle S_{p}, \psi_{p}^{\prime}\right\rangle$ and $S^{\prime}$ such that

(C1) $\quad S^{\prime} \subseteq S$

(C2) $\psi_{p}^{\prime}\left|S^{\prime} \subseteq \psi_{p}\right| S^{\prime}$, and

(C3) $\mathcal{P}^{\prime}$ is $f$-tolerant from $S^{\prime}$ for $\Sigma$.

Soundness and completeness. We say that an algorithm for the synthesis problem is sound iff its output meets the constraints of the Problem Statement 4.1. We say that an algorithm for the synthesis problem is complete iff it finds a solution to the Problem Statement 4.1 iff there exists one.

\section{Adding Nonmasking Fault-Tolerance}

Algorithm sketch. Since a nonmasking program is not required to satisfy its safety specification in the presence of faults, it only suffices to provide bounded-time recovery from the fault-span $S_{p}-S$ to the invariant $S$. We develop a general procedure that adds bounded-time recovery to a given region graph from any arbitrary given state predicate $P$ to another state predicate $Q$ within $\delta$ time units (i.e., $P \mapsto \leq \delta Q$ ). Notice that bounded-time recovery from fault-span to the invariant can be formally defined by $\mathcal{R} \equiv\left(S_{p}-S\right) \mapsto \leq \delta S$. The algorithm has four main steps. First, we transform the region graph to a weighted directed graph (called MaxDelay digraph [24]), in which the length of a path from vertex $v_{s}$ to $v_{t}$ is equivalent to the maximum delay for reaching the region that corresponds to $v_{t}$ from the region that corresponds to $v_{s}$. We use this property to remove the computations that violate $P \mapsto \leq \delta Q$. To this end, in Step 2, we rank vertices of the MaxDelay digraph by simply applying an adjusted Dijkstra's shortest path algorithms. For instance, suppose that a computation starts from a state $\sigma_{0} \in P$. If a fault perturbs the program to a state $\sigma_{j}$ where "something" should be redone, the maximum delay of that computation to reach $Q$ is obviously increased. Hence, we adjust the length of the shortest path from $\sigma_{0}$ to $Q$ such that the amount of time wasted by every occurrence of faults is considered (cf. Figure 1). In Step 3, we include regions and edges whose rank is at most the required response time $\delta$. Then, in Step 4, we transform the synthesized MaxDelay digraph back into a region graph.

Construction of MaxDelay digraph. We now describe the procedure ConstructMaxDelayGraph that transforms a region graph to a MaxDelay digraph. The procedure takes a region graph $R(\mathcal{P})\left\langle S_{p}^{r}, \psi_{p}^{r}\right\rangle$ and a set $f^{r}$ of fault edges as input, and constructs a MaxDelay digraph $G\langle V, A\rangle$ as follows. Vertices of $G$ consist of the regions in $R(\mathcal{P})$.

Notation: We denote the weight of an $\operatorname{arc}\left(v_{0}, v_{1}\right)$ by $\operatorname{Weight}\left(v_{0}, v_{1}\right)$. Let $\gamma$ denote a bijection that maps each region $r \in S_{p}^{r}$ to its corresponding vertex in $G$; i.e., $\gamma(r)$ is a vertex of $G$ that represents region $r$ of $R(\mathcal{P})$. Also, let $\gamma^{-1}$ denote the inverse of $\gamma$; i.e., 
$\gamma^{-1}(v)$ is the region of $R(\mathcal{P})$ that corresponds to vertex $v$ in $V$. Let $\Gamma$ be a function that maps a region predicate in $R(\mathcal{P})$ to the corresponding set of vertices of $G$ and let $\Gamma^{-1}$ be its inverse. Finally, for a boundary region $r$ with respect to clock variable $x$, we denote the value of $x$ by $r . x$ (equal to some nonnegative integer in $\mathbb{Z}_{\geq 0}$ ).

Arcs of $G$ consist of the following:

- Arcs of weight 0 from $v_{0}$ to $v_{1}$, if $\gamma^{-1}\left(v_{0}\right) \rightarrow \gamma^{-1}\left(v_{1}\right)$ represents a jump transition in $R(\mathcal{P})$.

- Arcs of weight $c^{\prime}-c$, where $c, c^{\prime} \in \mathbb{Z}_{\geq 0}$ and $c^{\prime}>c$, from $v_{0}$ to $v_{1}$, if $\gamma^{-1}\left(v_{0}\right)$ and $\gamma^{-1}\left(v_{1}\right)$ are both boundary regions with respect to clock variable $x_{i}$, such that $\gamma^{-1}\left(v_{0}\right) \cdot x_{i}=c, \gamma^{-1}\left(v_{1}\right) \cdot x_{i}=c^{\prime}$, and there is a path in $R(\mathcal{P})$ from $\gamma^{-1}\left(v_{0}\right)$ to $\gamma^{-1}\left(v_{1}\right)$, which does not reset $x_{i}$.

- Arcs of weight $c^{\prime}-c-\epsilon$, where $c, c^{\prime} \in \mathbb{Z}_{\geq 0}, c^{\prime}>c$, and $0<\epsilon \ll 1$, from $v_{0}$ to $v_{1}$, if (1) $\gamma^{-1}\left(v_{0}\right)$ is a boundary region with respect to clock $x_{i},(2) \gamma^{-1}\left(v_{1}\right)$ is an open region whose time-successor $\gamma^{-1}\left(v_{2}\right)$ is a boundary region with respect to clock $x_{i}$, (3) $\gamma^{-1}\left(v_{0}\right) \rightarrow \gamma^{-1}\left(v_{1}\right)$ represents a delay transition in $R(\mathcal{P})$, and (4) $\gamma^{-1}\left(v_{0}\right) \cdot x_{i}=c$ and $\gamma^{-1}\left(v_{2}\right) \cdot x_{i}=c^{\prime}$.

- Self-loop arcs of weight $\infty$ at vertex $v$, if $\gamma^{-1}(v)$ is an end region.

In order to compute the maximum delay between regions in $P^{r}$ and $Q^{r}$, it suffices to find the longest distance between $\Gamma\left(P^{r}\right)$ and $\Gamma\left(Q^{r}\right)$ in $G$.

We now describe the procedure Add_BoundedRecovery (cf. Figure 2) in detail. Given a region graph $R(\mathcal{P})$, we first transforms it into a MaxDelay digraph $G\langle V, A\rangle$ (Line A1). Recall that, by Assumption 3.2, faults are detectable and $\mathcal{P}$ has a variable that shows how many faults have occurred in a computation. Thus, let $G^{i}\left\langle V^{i}, A^{i}\right\rangle$ be the portion of $G$, in which $n-i$ faults have occurred, where $0 \leq i \leq n$. More specifically, initially, a computation starts from the portion $G^{n}$ where no faults have occurred. If a fault occurs in a computation that is currently in portion $G^{i}$, the computation will proceed in portion $G^{i-1}$. We use these portions to see whether it is possible to reach a vertex in $\Gamma\left(Q^{r}\right)$ from each vertex in $\Gamma\left(P^{r}\right)$ within $\delta$ time units.

Next, we rank vertices of all portions of $G$ using a modified Dijkstra's shortest path algorithm, which takes state perturbations into account (lines A2-A9 and A22A23). More specifically, since no faults occur in $G^{0}$, we first let the rank of each vertex $v \in V^{0}$ be the length of Dijkstra's shortest path from $v$ to $\Gamma\left(Q^{r}\right)^{0}$ (Line A2). Now, let $v_{0}$ be a vertex in $V^{i}$ where $1 \leq i \leq n$, and let $v_{1}$ be a vertex in $V^{i-1}$, such that

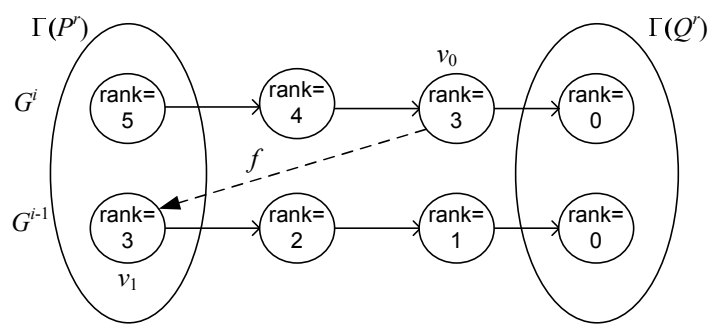

Fig. 1. Adjusted shortest path. 


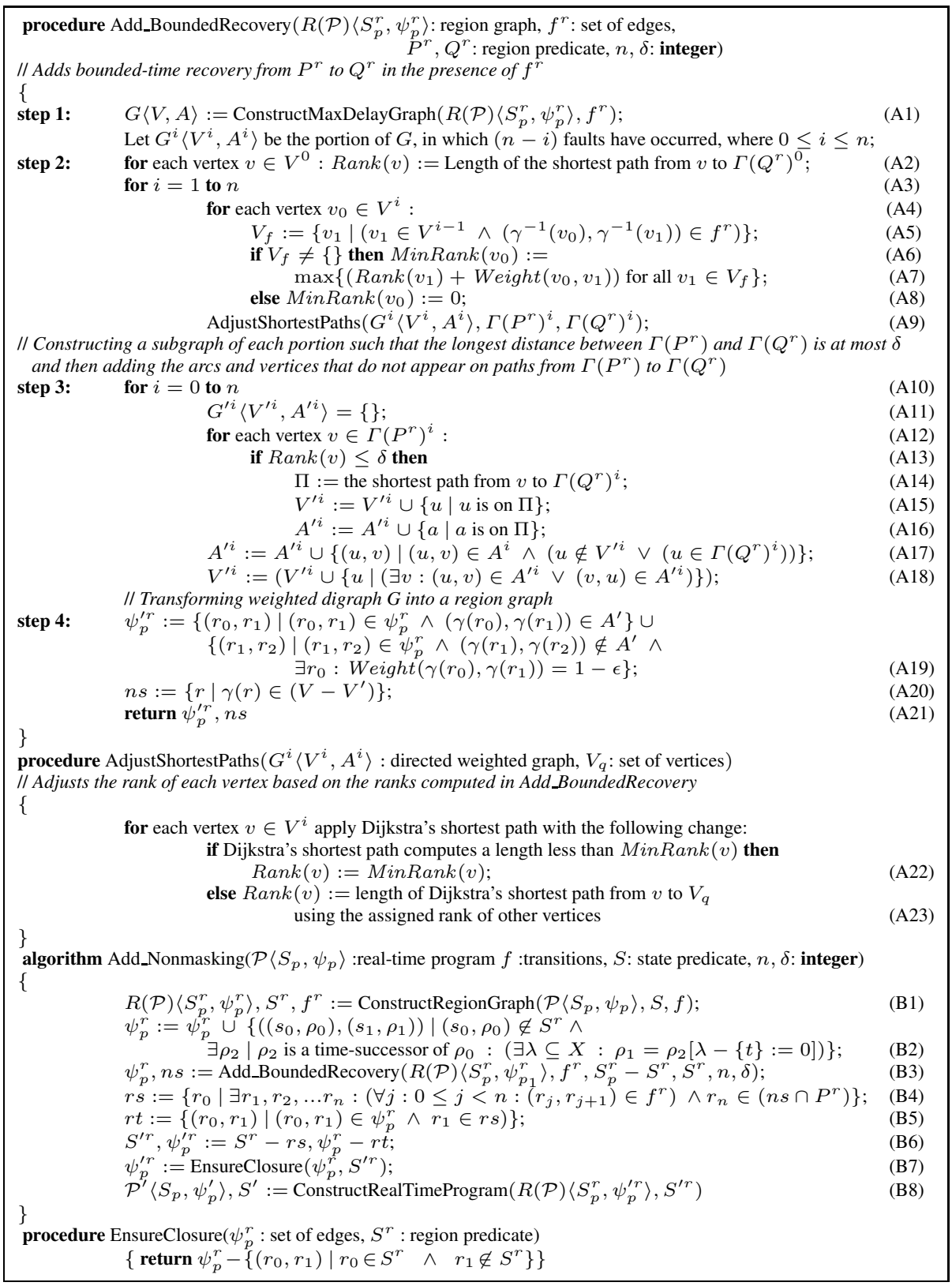

Fig. 2. Adding Bounded-Time Recovery/Nonmasking Fault-Tolerance. 
$\left(\gamma^{-1}\left(v_{0}\right), \gamma^{-1}\left(v_{1}\right)\right)$ is a fault edge in $R(\mathcal{P})$ and both $v_{0}$ and $v_{1}$ are on a path from $\Gamma\left(P^{r}\right)$ to $\Gamma\left(Q^{r}\right)$. There exist two cases: (1) the fault edge $\left(\gamma^{-1}\left(v_{0}\right), \gamma^{-1}\left(v_{1}\right)\right)$ decreases or does not change the computation delay, i.e, the shortest distance from $v_{1}$ to $\Gamma\left(Q^{r}\right)^{i-1}$ is less than or equal to the shortest distance from $v_{0}$ to $\Gamma\left(Q^{r}\right)^{i}$, and (2) the fault edge $\left(\gamma^{-1}\left(v_{0}\right), \gamma^{-1}\left(v_{1}\right)\right)$ increases the computation delay, i.e., the shortest distance from $v_{1}$ to $\Gamma\left(Q^{r}\right)^{i-1}$ is greater than the shortest distance from $v_{0}$ to $\Gamma\left(Q^{r}\right)^{i}$ (cf. Figure 1 for an example). While the former case does not cause violation of $P \mapsto \leq \delta Q$ in the presence of faults, the later may do. Hence, the rank of $v_{0} \in V^{i}$ must be at least the rank of $v_{1} \in V^{i-1}$. Moreover, if there exist multiple fault edges at $\gamma^{-1}\left(v_{0}\right)$ then we take the maximum rank (Line A7). After computing the rank of vertices from where faults may occur, we adjust the rank of the rest of vertices from where faults do not occur by invoking the procedure AdjustShortestPath (Line A9).

Now, for each portion $G^{i}$, we construct a subgraph of $G^{i}$ whose longest distance from each vertex in $\Gamma\left(P^{r}\right)^{i}$ to $\Gamma\left(Q^{r}\right)^{i}$ is at most $\delta$ as follows (lines A11-A16). We begin with an empty digraph $G^{\prime i}\left\langle V^{\prime i}, A^{\prime i}\right\rangle$ and we first include the shortest paths from each vertex $v \in \Gamma\left(P^{r}\right)^{i}$ to $\Gamma\left(Q^{r}\right)^{i}$, provided $\operatorname{Rank}(v) \leq \delta$ (lines A13-A16). Next, we include the remaining arcs and vertices in $G^{i}$, so that no arcs of the form $\left(v_{0}, v_{1}\right)$, where $v_{0}$ is on a path from $\Gamma\left(P^{r}\right)^{i}$ to $\Gamma\left(Q^{r}\right)^{i}$ are added (lines A17-A18).

Now, we transform the digraph $G^{\prime}$ back into a region graph (Line A19). Finally, we return the set $\psi_{p}^{\prime r}$ of edges from where $P \mapsto \leq \delta Q$ is not violated even in the presence of faults, and the set $n s$ of regions from where $P \mapsto \leq \delta Q$ may be violated in the presence of faults (lines A20-A21).

Using Add_BoundedRecovery to Add Nonmasking Fault-Tolerance. In order to add nonmasking fault-tolerance with bounded-time recovery $\delta$, we first transform the real-time program $\mathcal{P}\left\langle S_{p}, \psi_{p}\right\rangle$, invariant $S$, and the set of fault transitions $f$ into a region graph $R(\mathcal{P})\left\langle S_{p}^{r}, \psi_{p}^{r}\right\rangle$, region invariant $S^{r}$, and fault edges $f^{r}$ by invoking the procedure ConstructRegionGraph (Line B1), as described in Subsection 2.3. Next, in order to ensure that $S^{\prime}$ is reachable from all the states in $S_{p}-S^{\prime}$, we add recovery edges that start from each region in $S_{p}^{r}-S^{r}$ and go to regions where the time monotonicity condition is preserved, i.e., time is not decreased (Line B2). Notice that the algorithm allows arbitrary clock resets (except the clock that keeps track of the recovery time time $\delta$ ) during recovery, which is fine according to the definition of nonmasking fault-tolerance (such "new" clock resets occur only in states outside the invariant). Then we invoke the procedure Add_BoundedRecovery. This invocation identifies the set $r s$ of regions and the set $r t$ of edges from where faults alone may violate $\mathcal{R}$ (lines B4-B5). Then, it removes such regions (respectively, edges) from $S^{r}$ (respectively, $\psi_{p}^{r}$ ). Finally, the algorithm ensures the closure of the invariant (Line B7) and transforms the synthesized region graph $R\left(\mathcal{P}^{\prime}\right)$ back to a real-time program $\mathcal{P}^{\prime}$ (Line $\mathrm{B} 8$ ).

\section{Adding Soft-Failsafe Fault-Tolerance}

As mentioned in Subsection 2.2, the safety specification identifies a set $\Sigma_{b t}$ of bad transitions and a conjunction $\Sigma_{b r}$ of multiple bounded response properties. Also, recall that in the presence of faults, a soft-failsafe program is required to maintain $\Sigma_{b t}$ only. 
Algorithm sketch. We adapt the proposed algorithm in [6], which adds failsafe faulttolerance to untimed programs. Intuitively, our algorithm, consists of three main steps. First, we prohibit the program from reaching the set $m s$ of states from where a sequence of faults takes the program to a state where safety $\left(\Sigma_{b t}\right)$ is violated. Since our goal is to synthesize a maximal program, we find $m s$ by computing the smallest fixpoint of states from where safety may be violated. In step 2 , after removing $m s$ from the program invariant $S$, we make sure that this removal do not create new finite computations in the absence of faults. To this end, we remove deadlock states from the invariant which is in turn computing the largest fixpoint of the invariant. Finally, in step 3, we ensure that removal of transitions from where safety may be violated does not violate the closure of the output program.

We now describe the algorithm Add_SoftFalisafe (cf. Figure 3). We first transform the program $\mathcal{P}$ into its region graph $R(\mathcal{P})$ (Line $\mathrm{C} 1$ ). Then, the algorithm adds failsafe fault-tolerance to $R(\mathcal{P})$, so that no edge of $\Sigma_{b t}^{r}$ occurs in computations of $R(\mathcal{P})$ in the presence of faults by invoking the procedure Add_UntimedFailsafe (Line C2). This procedure first finds the set $m s$ of regions and the set $m t$ of edges from where safety of $\mathcal{P}$ may be violated by faults alone (lines E1-E2). Next, it removes such regions (respectively, edges) from the region invariant $S^{r}$ (respectively, set of edges $\psi_{p}^{r}$ ) of $R(\mathcal{P})$. Then, it removes deadlock regions from $S^{r}$ (Line E3), ensures the closure of $\psi_{p}^{r}$ in $S^{r}$ (Line E5), and returns a failsafe region graph $R\left(\mathcal{P}^{\prime}\right)\left\langle S_{p}^{r}, \psi_{p}^{\prime r}\right\rangle$ (Line E6). Finally, we transform the region graph $R\left(\mathcal{P}^{\prime}\right)$ back into a real-time program $\mathcal{P}^{\prime}$ (Line C3) as described in Subsection 2.3.

\section{Adding Hard-Failsafe Fault-Tolerance with one Bounded Response Property}

In this section, we present our algorithm Add_HardFailsafe (cf. Figure 3) for the case where the synthesized hard-failsafe program is required to satisfy at most one bounded response property in the presence of faults, i.e., $\Sigma_{b r} \equiv P \mapsto \leq \delta Q$.

Algorithm sketch. Intuitively, the algorithms works in five main steps. First, we add soft-failsafe to $R(\mathcal{P})$ to ensure that a transition in $\Sigma_{b t}$ occurs in no computation of $\mathcal{P}^{\prime}$. Note that, the outcome of adding soft-failsafe is a maximal program and every transition that is removed by Add_SoftFailsafe has to be removed, i.e., such transitions cannot be in any fault-tolerant program that satisfies the constraints of Problem Statement 4.1. In Step 2, we remove the behaviors that violate the bounded response property $\Sigma_{b r} \equiv$ $P \mapsto \leq \delta Q$ in the presence of faults using the procedure Add_BoundedRecovery. In step 3, we remove deadlock states due to removal of states and transitions in step 2. In Step 4, if a state $\sigma_{1} \in Q$ is removed and some state, say $\sigma_{0}$, in $P$ uses $\sigma_{1}$ to satisfy $P \mapsto \leq \delta Q$ then another path from $\sigma_{0}$ must be provided to satisfy $P \mapsto \leq \delta Q$. Hence, we remove $\sigma_{1}$ from $Q$ and repeat steps 2, 3, and 4 until no such $Q$-states exist. Finally, in Step 5, we ensure the closure of the output program.

We now describe the pseudo-code of the algorithm. In order to ensure that $\mathcal{P}^{\prime}$ maintains $\Sigma_{b t}$, we first add soft-failsafe fault-tolerance to $R(\mathcal{P})$ (Line D1). Next, we transform $\mathcal{P}$ into its region graph $R(\mathcal{P})$ (Line $\mathrm{D} 2$ ). Next, we modify $R(\mathcal{P})$, such that any computation that starts from a region in $P^{r}$, reaches a region in $Q^{r}$ in at most $\delta$ time 


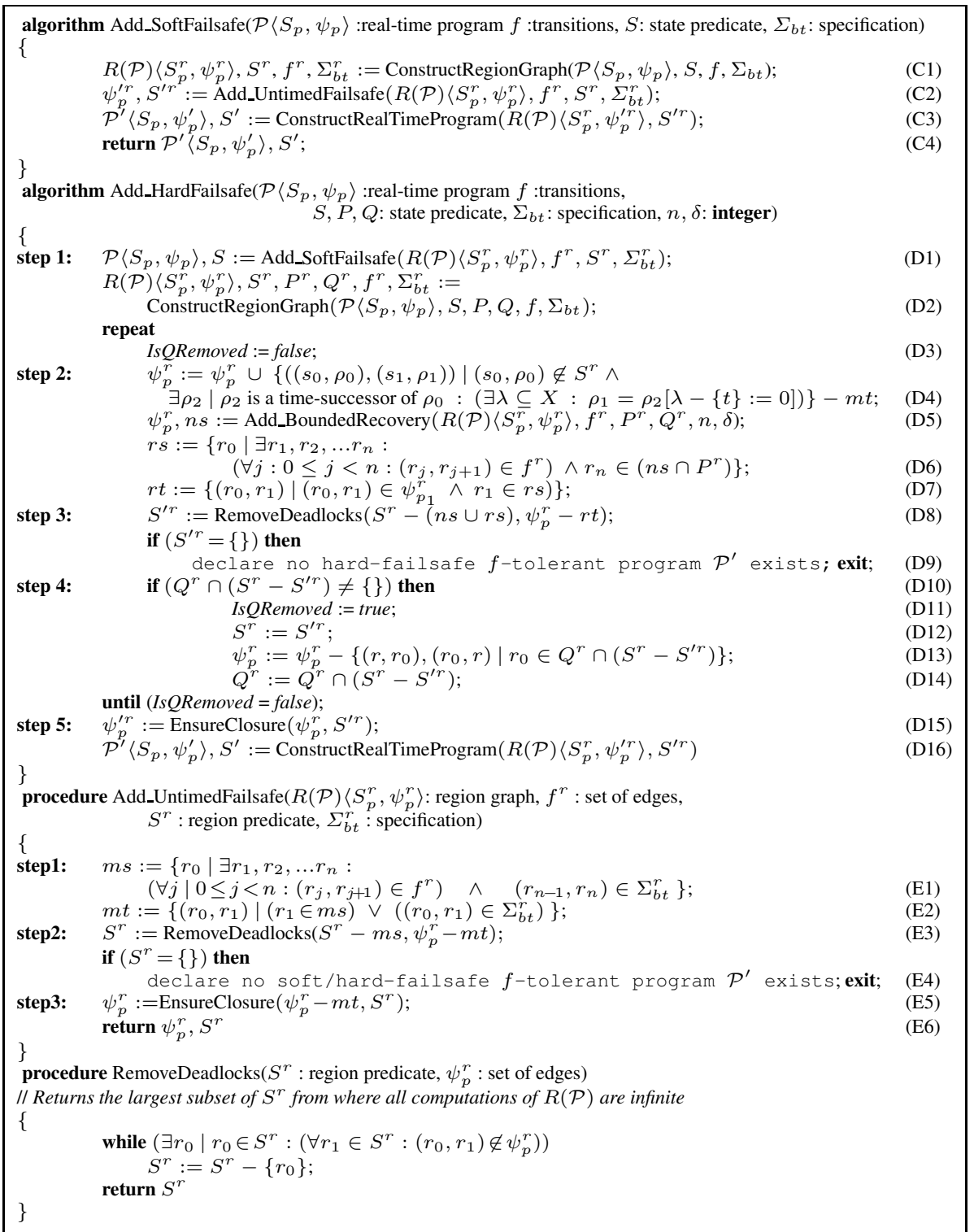

Fig. 3. Adding Failsafe Fault-Tolerance. 
units even in the presence of faults. Towards this end, we compute the set of regions and edges from where $\Sigma_{b r}$ is maintained (lines D3-D14). Precisely, in order to ensure that $Q$ is reachable from all the states in $P \wedge \neg S$, we first include edges that start from each region in $S_{p}^{r}-S^{r}$ and go to regions where the time monotonicity condition is preserved, i.e., time is not decreased (Line D4). Notice that the algorithm allows arbitrary clock resets as long as safety is not violated (by excluding the edges in $m t$ ). Then, we invoke the procedure Add_BoundedRecovery to ensure that $P \mapsto \leq \delta Q$ is maintained in the presence of faults (Line D5). Then, we identify the set $r s$ of regions and $r t$ of transitions from where $\Sigma_{b r}$ may be violated (lines D6-D7). We remove such regions and edges along with the deadlock regions from $S^{r}$ in the same fashion that we did for adding soft-failsafe (Line D8). However, we need to consider a special case where a region, say $r_{1}$, in $Q^{r}$ becomes a deadlock region. In this case, it is possible that all the regions along the paths that start from a region, say $r_{0}$, in $P^{r}$ and end in $r_{1}$ become deadlock regions. Hence, we need to find another path from $r_{0}$ to a region in $Q^{r}$ other than $r_{1}$. Thus, in this case, we remove $r_{1}$ (and similar regions) from $S^{r}$ and $Q^{r}$ and start over (lines D10-D14). Finally, the algorithm ensures closure of the invariant (Line $\mathrm{D} 15)$ and transforms the synthesized region graph $R\left(\mathcal{P}^{\prime}\right)$ back to a real-time program $\mathcal{P}^{\prime}$ (Line D16).

Theorem 7.1. The algorithms Add_Nonmasking and Add_Soft/HardFalisafe are sound and complete.

Theorem 7.2. The problem of adding nonmasking and soft/hard-failsafe fault-tolerance to a real-time program, where the synthesized program is required to satisfy at most one bounded response property in the presence of faults, is PSPACE-complete in the size of the input program.

\section{Conclusion}

In this paper, we focused on the problem of automatic addition of fault-tolerance to real-time programs. We considered three levels of fault-tolerance, namely failsafe, nonmasking, and masking. For failsafe and masking, we proposed two cases, soft and hard, based on satisfaction of timing constraints in the presence of faults. We first introduced a generic framework to formally define the notions of faults and fault-tolerance in the context of real-time programs. Then, we presented sound and complete algorithms for transforming fault-intolerant real-time programs into soft-failsafe and nonmasking fault-tolerant programs. We also proposed a sound and complete algorithm that synthesizes hard-failsafe fault-tolerant real-time programs, where the fault-tolerant program is required to satisfy at most one bounded response property in the presence of faults. The complexity of our algorithms are in polynomial time in the size region graphs. The results on synthesis of soft/hard-masking fault-tolerance are presented in a technical report [19].

\section{References}

1. M. Pandya and M. Malek. Minimum achievable utilization for fault-tolerant processing of periodic tasks. IEEE Transations on Computers, 47(10):1102-1112, 1998. 
2. D. Mossé, R. G. Melhem, and S. Ghosh. A nonpreemptive real-time scheduler with recovery from transient faults and its implementation. IEEE Transactions on Software Engineering, 29(8):752-767, 2003.

3. B. Bonakdarpour and S. S. Kulkarni. Automated incremental synthesis of timed automata. In International Workshop on Formal Methods for Industrial Critical Systems (FMICS), 2006.

4. K. M. Chandy and J. Misra. Parallel Program Design: A Foundation. Addison-Wesley, 1988.

5. A. Ebnenasir, S. S. Kulkarni, and B. Bonakdarpour. Revising UNITY programs: Possibilities and limitations. In 9th International Conference on Principles of Distributed Systems (OPODIS), 2005.

6. S. S. Kulkarni and A. Arora. Automating the addition of fault-tolerance. In Formal Techniques in Real-Time and Fault-Tolerant Systems (FTRTFT), pages 82-93, 2000.

7. S. S. Kulkarni, A. Arora, and A. Chippada. Polynomial time synthesis of Byzantine agreement. In 20th Symposium on Reliable Distributed Systems (SRDS), pages 130-140, 2001.

8. S. S. Kulkarni and A. Ebnenasir. Automated synthesis of multitolerance. In International Conference on Dependable Systems and Networks (DSN), pages 209-219, 2004.

9. P. C. Attie, A. Arora, and E. A. Emerson. Synthesis of fault-tolerant concurrent programs. ACM Transactions on Programming Languages and Systems, 26(1):125-185, 2004.

10. E. Asarin, O. Maler, A. Pnueli, and J. Sifakis. Controller synthesis for timed automata. In IFAC Symposium on System Structure and Control, pages 469-474, 1998.

11. E. Asarin and O. Maler. As soon as possible: Time optimal control for timed automata. In Hybrid Systems: Computation and Control (HSCC), pages 19-30, 1999.

12. D. D'Souza and P. Madhusudan. Timed control synthesis for external specifications. In 19th Annual Symposium on Theoretical Aspects of Computer Science (STACS), pages 571-582, 2002.

13. P. Bouyer, D. D'Souza, P. Madhusudan, and A. Petit. Timed control with partial observability. In Computer Aided Verification (CAV), pages 180-192, 2003.

14. L. de Alfaro, M. Faella, T. A. Henzinger, R. Majumdar, and M. Stoelinga. The element of surprise in timed games. In 14th International Conference on Concurrency Theory (CONCUR), 2003.

15. M. Faella, S. LaTorre, and A. Murano. Dense real-time games. In Logic in Computer Science (LICS), pages 167-176, 2002.

16. R. Alur, C. Courcoubetis, and D. Dill. Model-checking in dense real-time. Information and Computation, 104(1):2-34, 1993.

17. R. Alur and D. Dill. A theory of timed automata. Theoretical Computer Science, 126(2):183235, 1994.

18. Y. Abdeddam. Scheduling with Timed Automata. PhD thesis, INPG, Grenoble, November 2002.

19. B. Bonakdarpour and S. S. Kulkarni. Automatic addition of fault-tolerance to real-time programs. Technical Report MSU-CSE-06-13, Department of Computer Science and Engineering, Michigan State University, 2006.

20. R. Alur and T. A. Henzinger. Real-time system = discrete system + clock variables. International Journal on Software Tools for Technology Transfer, 1(1-2):86-109, 1997.

21. T. A. Henzinger. Sooner is safer than later. Information Processing Letters, 43(3):135-141, 1992.

22. B. Alpern and F. B. Schneider. Defining liveness. Information Processing Letters, 21:181$185,1985$.

23. A. Arora and M. G. Gouda. Closure and convergence: A foundation of fault-tolerant computing. IEEE Transactions on Software Engineering, 19(11):1015-1027, 1993.

24. C. Courcoubetis and M. Yannakakis. Minimum and maximum delay problems in real-time systems. In Computer-Aided Verificaion (CAV), pages 399-409, 1991. 